\title{
A framework for optimizing micro-CT in dual-modality micro-CT/XFCT small-animal imaging system
}

Srinivasan Vedantham, Suman Shrestha, Andrew Karellas, Sang Hyun Cho

Srinivasan Vedantham, Suman Shrestha, Andrew Karellas, Sang Hyun Cho, "A framework for optimizing micro-CT in dual-modality micro-CT/XFCT small-animal imaging system," Proc. SPIE 10393, Radiation Detectors in Medicine, Industry, and National Security XVIII, 103930R (7 September 2017); doi: 10.1117/12.2279351

SPIE Event: SPIE Optical Engineering + Applications, 2017, San Diego, California, United States 


\title{
A framework for optimizing micro-CT in dual-modality micro- CT/XFCT small-animal imaging system
}

\author{
Srinivasan Vedantham*a, Suman Shrestha ${ }^{\mathrm{b}}$, Andrew Karellas ${ }^{\mathrm{a}}$, Sang Hyun $\mathrm{Cho}^{\mathrm{c}}$ \\ ${ }^{a}$ Department of Medical Imaging, University of Arizona, Tucson, AZ, USA 85724; \\ ${ }^{\mathrm{b}}$ Department of Radiology, University of Massachusetts Medical School, Worcester, MA, USA \\ 01655; ${ }^{\mathrm{c}}$ Department of Radiation Physics, The University of Texas MD Anderson Cancer Center, \\ Houston, TX, USA 77030
}

\begin{abstract}
Dual-modality Computed Tomography (CT)/X-ray Fluorescence Computed Tomography (XFCT) can be a valuable tool for imaging and quantifying the organ and tissue distribution of small concentrations of high atomic number materials in small-animal system. In this work, the framework for optimizing the micro-CT imaging system component of the dualmodality system is described, either when the micro-CT images are concurrently acquired with XFCT and using the $\mathrm{x}$ ray spectral conditions for XFCT, or when the micro-CT images are acquired sequentially and independently of XFCT. This framework utilizes the cascaded systems analysis for task-specific determination of the detectability index using numerical observer models at a given radiation dose, where the radiation dose is determined using Monte Carlo simulations.
\end{abstract}

Keywords: X-ray fluorescence computed tomography (XFCT), computed tomography (CT), small-animal imaging system

\section{INTRODUCTION}

Several targeted nanoparticles incorporating high atomic number $(\mathrm{Z})$ elements such as gold $(\mathrm{Au})$, silver $(\mathrm{Ag})$ and iodine (I) have been developed and are actively investigated for potential use as diagnostic contrast agents, as therapeutic agents for targeted treatment, and as theranostic agents [1-3]. These nanoparticle-based agents are initially evaluated in preclinical small-animal models to evaluate safety, biodistribution and suitability for subsequent clinical trials. For such studies in preclinical models, there is a need for in vivo imaging of the nanoparticle distribution as an alternative to tissue analysis following euthanasia. Such an imaging system can also allow for monitoring temporal changes of the nanoparticle distribution. X-ray fluorescence computed tomography (XFCT) has the potential to serve this important need [4-8]. For example, benchtop XFCT utilizing a conventional polychromatic x-ray source has shown to be capable of imaging very low concentration of gold nanoparticles (GNPs) present in a mouse on the order of $0.2 \%$ to $2 \%$ by weight [8]. While XFCT provides for the spatial distribution and allows quantitation of the high $\mathrm{Z}$ element concentration, it does not provide anatomical images. A micro-computed tomography (CT) system used in conjunction with benchtop XFCT can allow for co-localization of the anatomy with the spatial distribution of the nanoparticle agent that incorporates a high $\mathrm{Z}$ element.

The motivation for this study was that the radiation dose from benchtop XFCT can be in the range of 15 to 20 cGy [7] and is comparable to the radiation dose from micro-CT reported in some studies (e.g., [9]). Hence, a dual-modality micro-CT/XFCT small-animal imaging system has the potential to double the radiation dose. In order to limit the radiation dose, the potential for concurrently acquiring the micro-CT images using the XFCT x-ray beam was investigated. A major challenge is that the x-ray spectrum used for benchtop XFCT is substantially different with $\mathrm{x}$-ray tube peak voltage as high as $125 \mathrm{kV}$ and with heavily filtration using tin ( $\mathrm{Sn})$ or lead $(\mathrm{Pb})$ to induce gold $\mathrm{K}$-shell $\mathrm{x}$-ray fluorescence from GNPs. In comparison, micro-CT operates in the 40-90 kV range [9, 10]. The frame-work presented in here can be readily adapted for both concurrent and sequential micro-CT imaging with benchtop XFCT at a specified radiation dose. The frame-work can also facilitate task-dependent optimization of image acquisition factors in existing micro-CT systems.

*svedantham@radiology.arizona.edu; phone $1 \quad 520 \quad 626-6641 ; \quad$ fax $1 \quad 520$ 626-7093; medicine.arizona.edu

Radiation Detectors in Medicine, Industry, and National Security XVIII, edited by Gary P. Grim,

Lars R. Furenlid, H. Bradford Barber, Proc. of SPIE Vol. 10393, 103930R · @ 2017 SPIE

CCC code: $0277-786 \mathrm{X} / 17 / \$ 18 \cdot$ doi: $10.1117 / 12.2279351$ 


\section{METHODS}

\subsection{Modeling approach}

The modeling approach comprises four major components. First, for a specified imaging geometry and small-animal model, Monte Carlo simulations are used to estimate the radiation dose to the small-animal. Second, for specified imaging geometry, detector specifications and acquisition parameters selected to satisfy the radiation dose limit, cascaded systems analysis is used to determine the objective physics-based imaging metrics such as modulation transfer function (MTF), noise power spectrum (NPS), noise-equivalent quanta (NEQ), and detective quantum efficiency (DQE). Third, an imaging task is specified for task-specific evaluation. Fourth, numerical observer models are then used to determine the detectability index by combining the task function with objective imaging properties.

\subsection{Imaging geometry}

The distance from the axis of rotation, also referred to as isocenter, to the source for most micro-CT systems are in the range of 100-200 mm. The imaging geometry reported for a cone-beam micro-CT system [10] was used and this conebeam micro-CT scanner is denoted as the reference system. The source-to-isocenter distance (SCD) and the source to detector distance (SDD) were $154 \mathrm{~mm}$ and $265 \mathrm{~mm}$, respectively. This system was assumed to use a PaxScan 1515 (Varex Imaging, Salt Lake City, UT) amorphous silicon flat-panel detector with $200 \mu \mathrm{m}$ thick CsI:Tl scintillator. X-ray source, detector and acquisition parameters are summarized in Table 1 for this reference system. For the dual-modality micro-CT/XFCT system, $\mathrm{SCD}=145 \mathrm{~mm}$ and $\mathrm{SDD}=250 \mathrm{~mm}$ were used for simulations, which slightly differs from the $\mathrm{SDD}=240 \mathrm{~mm}$ for the benchtop system. An active-pixel complementary metal-oxide-semiconductor (CMOS) detector (Dexela 1512, Varex Imaging, Salt Lake City, UT) with $150 \mu \mathrm{m}$ thick CsI:Tl scintillator was considered based on our current experimental setup. This scanner is referred to as "mCT/XFCT" system with a prefix of "C" or " $\mathrm{S}$ ", representing concurrent or sequential micro-CT imaging. The x-ray source, detector and acquisition parameters for the XFCT system are also included in Table 1.

Table 1. Acquisition parameters, x-ray source and detector specifications for reference cone-beam micro-CT system, concurrent micro-CT/XFCT (C-mCT/XFCT) imaging using the XFCT source and sequential micro-CT/XFCT (S$\mathrm{mCT} / \mathrm{XFCT}$ ) imaging.

\begin{tabular}{|c|c|c|c|}
\hline Parameters/Specifications & Reference & C-mCT/XFCT & S-mCT/XFCT \\
\hline \multicolumn{4}{|l|}{ Imaging geometry } \\
\hline Source-to-isocenter distance (SCD) & $154 \mathrm{~mm}$ & $145 \mathrm{~mm}$ & $145 \mathrm{~mm}$ \\
\hline Source-to-detector distance (SDD) & $265 \mathrm{~mm}$ & $250 \mathrm{~mm}$ & $250 \mathrm{~mm}$ \\
\hline \multicolumn{4}{|l|}{ X-ray source } \\
\hline Focal spot & $5 \mu \mathrm{m}$ & $400 \mu \mathrm{m}$ & $400 \mu \mathrm{m}$ \\
\hline KV & 90 & 125 & 90 \\
\hline Anode & $\mathrm{W}$ & $\mathrm{W}$ & $\mathrm{W}$ \\
\hline Be window & $0.15 \mathrm{~mm}$ & $0.8 \mathrm{~mm}$ & $0.8 \mathrm{~mm}$ \\
\hline $\mathrm{X}$-ray beam filtration & $\begin{array}{c}0.06 \mathrm{~mm} \mathrm{Cu}+ \\
0.5 \mathrm{~mm} \mathrm{Al} \\
\end{array}$ & $2 \mathrm{~mm} \mathrm{Sn}$ & $\begin{array}{c}0.06 \mathrm{~mm} \mathrm{Cu}+ \\
0.5 \mathrm{~mm} \mathrm{Al} \\
\end{array}$ \\
\hline \multicolumn{4}{|l|}{ Detector } \\
\hline Pixel pitch & $127 \mu \mathrm{m}$ & $75 \mu \mathrm{m}$ & $75 \mu \mathrm{m}$ \\
\hline Fill factor & 0.57 & 0.84 & 0.84 \\
\hline CsI:Tl thickness & $200 \mu \mathrm{m}$ & $150 \mu \mathrm{m}$ & $150 \mu \mathrm{m}$ \\
\hline System noise & 3945 e- & 362 e- & 362 e- \\
\hline \multicolumn{4}{|l|}{ Acquisition parameters } \\
\hline Number of projections & 512 & 64 & 512 \\
\hline Dose limit & 16 mGy & $150 \mathrm{mGy}$ & $16 \mathrm{mGy}$ \\
\hline Reconstruction & FBP (ramp) & FBP (ramp) & FBP (ramp) \\
\hline
\end{tabular}




\subsection{Monte Carlo simulations}

Monte Carlo simulations were performed using the GEANT4 toolkit using the Livermore low-energy physics model. For x-ray photon interactions, photoelectric effect, Compton and Rayleigh scattering were considered similar to prior studies [11-15]. One million monoenergy x-ray photons from 10 to $125 \mathrm{keV}$, in $1 \mathrm{keV}$ increments were used in the simulation. The small-animal was approximated as a water-equivalent cylinder of $30 \mathrm{~mm}$ diameter and $100 \mathrm{~mm}$ length. Each $\mathrm{x}$-ray photon was tracked and the dose per incident $\mathrm{x}$-ray photon of specified energy was determined. For a given $\mathrm{x}$-ray spectrum, the aforementioned dose per incident photon was weighted after normalizing the spectrum to unit area. Figure 1 shows the $\mathrm{x}$-ray spectra for the reference cone-beam micro-CT system and that used for XFCT imaging.

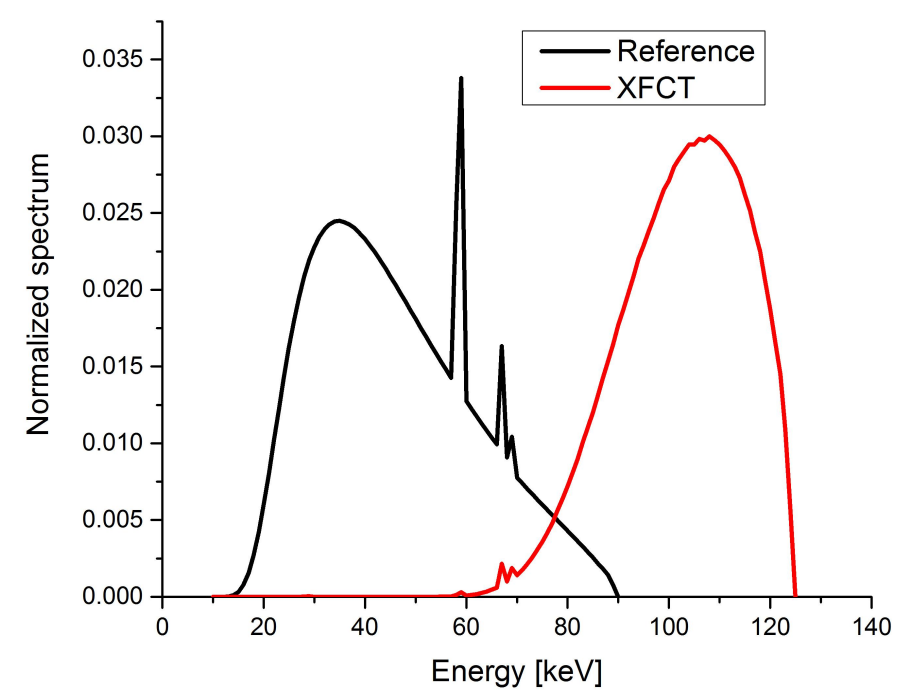

Figure 1. X-ray spectra used for imaging with reference cone-beam micro-CT system and XFCT. Each spectrum is normalized to unit area.

\subsection{Cascaded systems analysis}

Cascaded systems analysis that models the signal and noise transfer properties in the spatial frequency domain through each step of the image formation chain was used to obtain objective imaging metrics such as MTF, NPS, NEQ and DQE $[16,17]$. The specific implementation of the model accounted for emission of characteristic x-rays and reabsorption within the scintillator [18-20]. Such models have been well-validated in projection space and have been extended to reconstructed image space. For image reconstruction, ramp-filtered back projection without apodization and bilinear interpolation of voxels were modeled.

\subsection{Task-specific evaluation}

The purpose of the micro-CT component of the dual-modality micro-CT/XFCT imaging system is to provide anatomical images of the small animal. Hence, the tasks considered were detection of 0.5 to $3 \mathrm{~mm}$ diameter spheres with CT numbers ranging from 5 to $200 \mathrm{HU}$. In each simulation, the sphere was considered to be centered at the isocenter, defined as the intersection of the central-ray $\left(0^{\circ}\right.$ cone and fan angles) with the axis of rotation. At a specified dose limit (Table 1), the x-ray fluence integrated over the entire $360^{\circ}$ cone-beam scan was determined and equally divided among the projections. The parallel-cascades systems model was used to determine the objective imaging metrics in reconstructed object domain. The ICRU-recommended detectability index $(d)$ was computed by combining the imaging task with the objective imaging metrics using an ideal numerical observer model. 


\section{RESULTS AND DISCUSSION}

Figure 2 shows the radiation dose to the water-equivalent cylindrical phantom approximating a small animal for monoenergy x-ray photons from 10 to $125 \mathrm{keV}$ reported in units of dose in $\mu$ Gy per x-ray photon. The data are shown for SCD of 100 and $200 \mathrm{~mm}$ that spans the range of imaging geometries used in micro-CT systems. The slight increase in dose with the larger SCD is expected due to the longer path-length for oblique rays through the object. Upon weighting with the normalized 90 and $125 \mathrm{kV}$ spectra, the doses in $\mu$ Gy per $\mathrm{x}$-ray photon are $5.5 \times 10^{-7}$ and $5.4 \times 10^{-7}$, respectively.

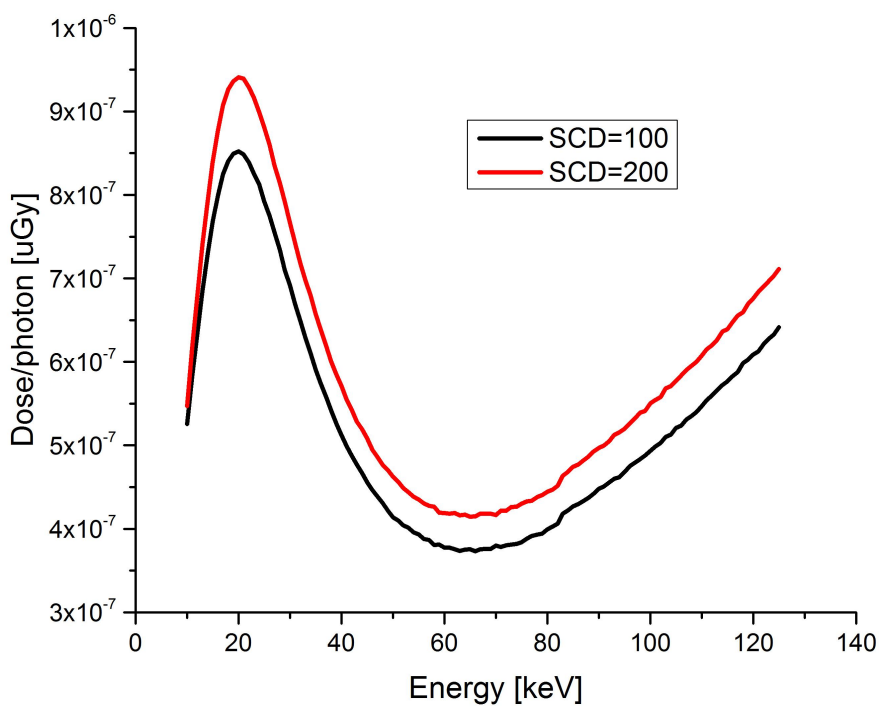

Figure 2. Radiation dose in $\mu$ Gy per incident $\mathrm{x}$-ray photon for monoenergy $\mathrm{x}$-rays for source-to-isocenter distances (SCD) of 100 and $200 \mathrm{~mm}$.

Figures 3A through $3 \mathrm{C}$ show the MTF for the reference system, sequential micro-CT (S-mCT/XFCT) imaging and concurrent (C-mCT/XFCT) imaging. In each plot, the following color-coding scheme is utilized: red - focal spot MTF inclusive of the system magnification associated blur, cyan - MTF associated with the emission and reabsorption of characteristic x-rays, green - scintillator blur, yellow - pixel aperture function, black - the projection space MTF that combines all of the above, blue - bilinear interpolation used during image reconstruction, and magenta - the MTF in the reconstructed object domain. In Figure 3A for the reference system, the focal spot MTF is close to unity over the entire spatial frequency range due to the use of a $5 \mu \mathrm{m}$ micro-focus $\mathrm{x}$-ray tube. The major contributor to the reduction in overall system MTF (magenta curve) in Fig. 3A is the scintillator associated blur. Comparing Figure 3A with Figure 3B, there is a reduction in limiting spatial resolution $(10 \% \mathrm{MTF})$ from 4.5 cycles $/ \mathrm{mm}$ for the reference system to 3.5 cycles/mm for sequential micro-CT imaging. Although the S-mCT/XFCT (Figure 3B) utilizes a thinner scintillator compared to the reference system $(150 \mu \mathrm{m}$ vs. $200 \mu \mathrm{m})$ and has a finer pixel pitch $(75 \mu \mathrm{m}$ vs. $127 \mu \mathrm{m})$, there is reduction in overall system MTF due to the substantially larger x-ray tube focal spot size ( $400 \mu \mathrm{m}$ vs. $5 \mu \mathrm{m}$ ). Comparing Figure 3B with Figure 3C, there is minimal difference in the limiting spatial resolution, as the major contributing factor to the overall MTF is the x-ray tube focal spot size. For concurrent micro-CT/XFCT (Figure 3C), the larger focal spot size is unavoidable as XFCT requires a focal spot capable of providing sufficient fluence rate to complete the exam in a reasonable duration. However, for sequential micro-CT/XFCT (Figure 3B), it is potentially possible to use a second xray tube with a smaller focal spot size for micro-CT imaging. Such an approach also provides flexibility for choosing the appropriate technique factors for micro-CT imaging without the constraints of XFCT imaging.

Figure 4 shows the detectability index computed using an ideal observer model for 0.5 to $3 \mathrm{~mm}$ diameter spheres with $\mathrm{CT}$ numbers ranging from 5 to $200 \mathrm{HU}$. The color-coding scheme for the curves represents objects (spheres) of diameters ranging from $500 \mu \mathrm{m}$ to $3 \mathrm{~mm}$ and is matched between the reference (Ref) system, sequential micro-CT (S$\mathrm{mCT})$ imaging and concurrent (C-mCT) imaging. Comparing the reference system to sequential micro-CT imaging, 
there is an improvement in detectability index, primarily due to the significantly lower noise characteristics of the CMOS sensor compared to the amorphous silicon detector (3945 e- vs. 362 e-). It is relevant to note that both these systems use $90 \mathrm{kV}$ spectrum and similar acquisition factors (Table 1) and are matched in terms of radiation dose to the small-animal. Comparing concurrent micro-CT/XFCT imaging (C-mCT) with the reference system and with sequential micro-CT/XFCT imaging, there is a significant improvement in the detectability index. This improvement in detectability index is due to an approximately ten-fold increase in radiation dose with concurrent micro-CT/XFCT imaging. It is relevant to note that this is not additional radiation dose to the animal.

3A: Reference system

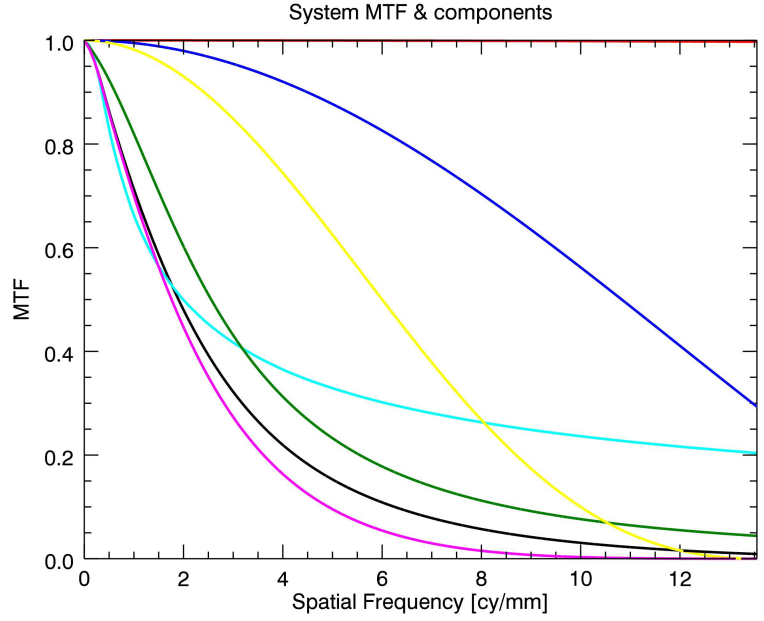

3B: S-mCT/XFCT system

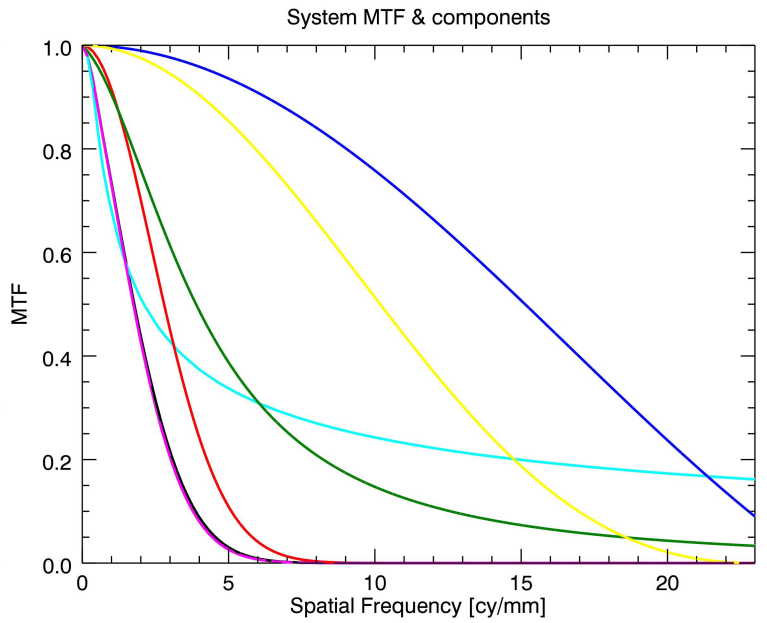

3C: $\mathrm{C}-\mathrm{mCT} / \mathrm{XFCT}$ system

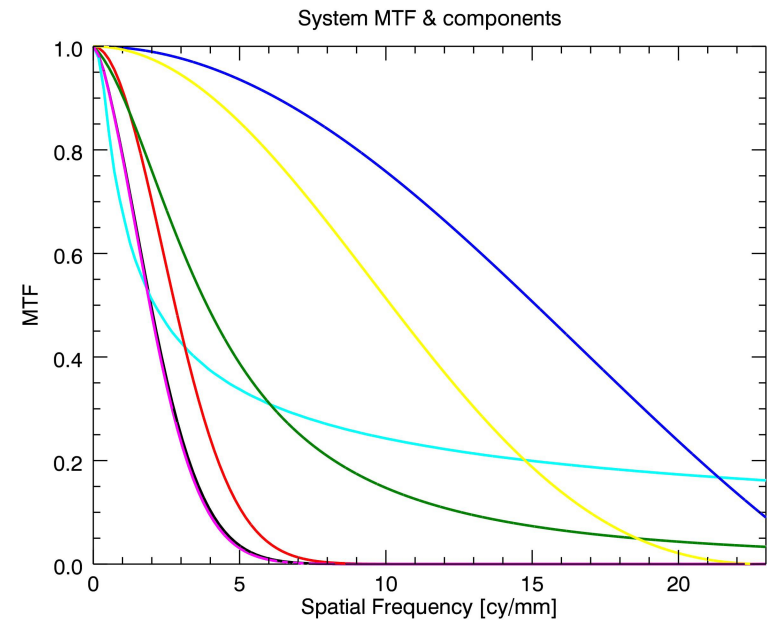

Figure 3. The overall system MTF along with the components of the imaging chain that contribute to the overall system MTF are shown for (a) the reference system, (b) for sequential micro-CT/XFCT imaging, and (c) for concurrent micro$\mathrm{CT} / \mathrm{XFCT}$ imaging. In each panel, the following color-coding is used: red - focal spot MTF inclusive of the system magnification associated blur, cyan - MTF associated with the emission and reabsorption of characteristic x-rays, green scintillator blur, yellow - pixel aperture function, black - the projection space MTF that combines all of the above, blue bilinear interpolation used during image reconstruction, and magenta - the MTF in the reconstructed object domain.

Computing the slope for each curve and comparing it between the reference system, concurrent, and sequential micro$\mathrm{CT} / \mathrm{XFCT}$ imaging shows that the slope for concurrent micro-CT/XFCT imaging is higher and increases with object diameter. This is readily supported by visual analysis of Figure 4. It can thus be inferred that concurrent micro- 
$\mathrm{CT} / \mathrm{XFCT}$ imaging is preferable when the objective of micro-CT imaging is to provide gross anatomical imaging of high-contrast structures such as the skeleton for co-localization with XFCT. However, if the intent is to visualize lowcontrast, high-resolution structures then sequential micro-CT imaging using an additional dedicated micro-focus x-ray tube, based on the observation in Figure 3, would be preferable.

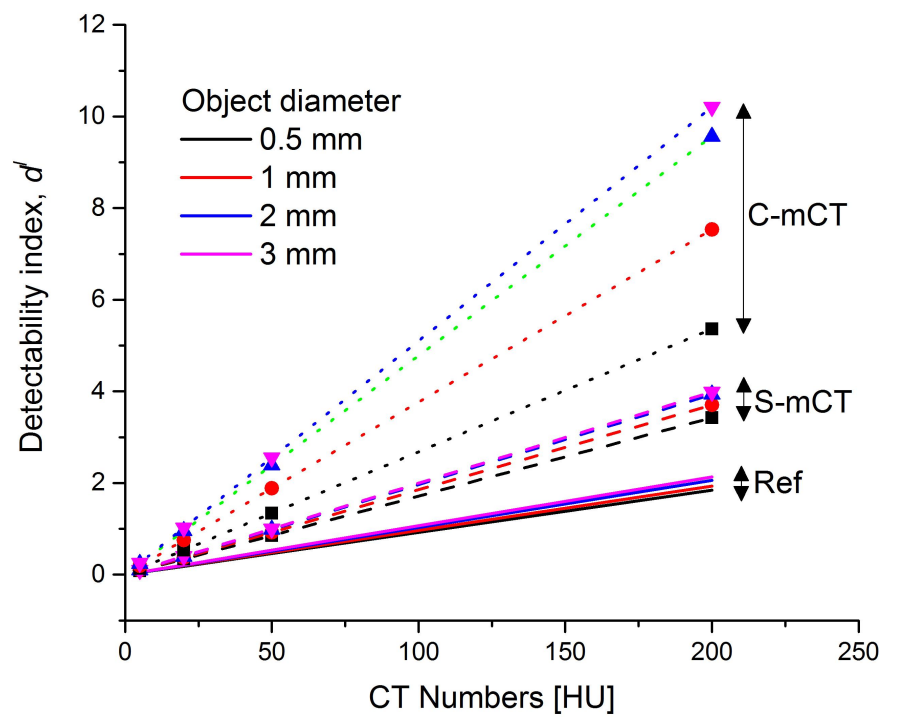

Figure 4. The detectability index computed using an ideal observer model for the reference system, denoted as "Ref", for sequential micro-CT/XFCT imaging, denoted as "S-mCT", and for concurrent micro-CT/XFCT imaging, denoted as "C-mCT".

\section{CONCLUSION}

The results from this study provide a clearer picture of the trade-off between concurrent and sequential micro-CT imaging in dual-modality micro-CT/XFCT. If the intent of the micro-CT imaging is to provide gross anatomical images for co-localization with XFCT, then concurrent micro-CT/XFCT imaging is preferable. However, if the intent of micro$\mathrm{CT}$ imaging is to provide high-resolution anatomical images similar to stand-alone micro-CT systems, then sequential micro-CT imaging with a micro-focus x-ray tube dedicated to micro-CT imaging would be of benefit.

\section{ACKNOWLEDGEMENTS}

This work was supported in part by National Institutes of Health (NIH) grants R01 EB020658, R01 CA195512 and R01 CA199044. The contents are solely the responsibility of the authors and do not reflect the official views of the NIH.

\section{REFERENCES}

[1] K. Sokolov, M. Follen, J. Aaron et al., "Real-time vital optical imaging of precancer using anti-epidermal growth factor receptor antibodies conjugated to gold nanoparticles," Cancer Research, 63(9), 1999-2004 (2003).

[2] S. H. Cho, B. L. Jones, and S. Krishnan, "The dosimetric feasibility of gold nanoparticle-aided radiation therapy (GNRT) via brachytherapy using low-energy gamma-/x-ray sources," Physics in Medicine \& Biology, 54(16), 4889 (2009).

[3] S. Krishnan, P. Diagaradjane, and S. H. Cho, "Nanoparticle-mediated thermal therapy: evolving strategies for prostate cancer therapy,” Int J Hyperthermia, 26(8), 775-89 (2010). 
[4] P. J. La Riviere, and P. A. Vargas, "Monotonic penalized-likelihood image reconstruction for X-ray fluorescence computed tomography," IEEE Trans Med Imaging, 25(9), 1117-29 (2006).

[5] S. K. Cheong, B. L. Jones, A. K. Siddiqi et al., "X-ray fluorescence computed tomography (XFCT) imaging of gold nanoparticle-loaded objects using 110 kVp x-rays," Phys Med Biol, 55(3), 647-62 (2010).

[6] M. Bazalova, Y. Kuang, G. Pratx et al., "Investigation of X-ray fluorescence computed tomography (XFCT) and K-edge imaging," IEEE Trans Med Imaging, 31(8), 1620-7 (2012).

[7] B. L. Jones, N. Manohar, F. Reynoso et al., "Experimental demonstration of benchtop x-ray fluorescence computed tomography (XFCT) of gold nanoparticle-loaded objects using lead- and tin-filtered polychromatic cone-beams," Phys Med Biol, 57(23), N457-67 (2012).

[8] N. Manohar, F. J. Reynoso, P. Diagaradjane et al., "Quantitative imaging of gold nanoparticle distribution in a tumor-bearing mouse using benchtop x-ray fluorescence computed tomography," Sci Rep, 6, 22079 (2016).

[9] A. L. Goertzen, A. K. Meadors, R. W. Silverman et al., "Simultaneous molecular and anatomical imaging of the mouse in vivo," Phys Med Biol, 47(24), 4315-28 (2002).

[10] N. Miyahara, T. Kokubo, Y. Hara et al., "Evaluation of X-ray doses and their corresponding biological effects on experimental animals in cone-beam micro-CT scans (R-mCT2)," Radiol Phys Technol, 9(1), 60-8 (2016).

[11] I. Sechopoulos, S. Suryanarayanan, S. Vedantham et al., "Computation of the glandular radiation dose in digital tomosynthesis of the breast," Med Phys, 34(1), 221-32 (2007).

[12] I. Sechopoulos, S. Vedantham, S. Suryanarayanan et al., "Monte Carlo and phantom study of the radiation dose to the body from dedicated CT of the breast," Radiology, 247(1), 98-105 (2008).

[13] I. Sechopoulos, S. Suryanarayanan, S. Vedantham et al., "Radiation dose to organs and tissues from mammography: Monte Carlo and phantom study," Radiology, 246(2), 434-43 (2008).

[14] S. Vedantham, L. Shi, A. Karellas et al., "Dedicated breast CT: radiation dose for circle-plus-line trajectory," Med Phys, 39(3), 1530-41 (2012).

[15] L. Shi, S. Vedantham, A. Karellas et al., "Library based x-ray scatter correction for dedicated cone beam breast CT,” Med Phys, 43(8), 4529 (2016).

[16] S. Vedantham, A. Karellas, and S. Suryanarayanan, "Solid-state fluoroscopic imager for high-resolution angiography: parallel-cascaded linear systems analysis," Med Phys, 31(5), 1258-68 (2004).

[17] D. J. Tward, and J. H. Siewerdsen, "Noise aliasing and the 3D NEQ of flat-panel cone-beam CT: effect of 2D/3D apertures and sampling," Med Phys, 36(8), 3830-43 (2009).

[18] S. Vedantham, A. Karellas, S. Suryanarayanan et al., "Solid-state fluoroscopic imager for high-resolution angiography: physical characteristics of an $8 \mathrm{~cm}$ x $8 \mathrm{~cm}$ experimental prototype," Med Phys, 31(6), 1462-72 (2004).

[19] S. Suryanarayanan, A. Karellas, S. Vedantham et al., "Theoretical analysis of high-resolution digital mammography," Phys Med Biol, 51(12), 3041-55 (2006).

[20] S. Vedantham, and A. Karellas, "Modeling the performance characteristics of computed radiography (CR) systems," IEEE Trans Med Imaging, 29(3), 790-806 (2010). 